\title{
Long-Chain Acyl-Coenzyme A Dehydrogenase Deficiency: Biochemical Studies in Fibroblasts from Three Patients ${ }^{1}$
}

\author{
BRAD A. AMENDT, ANN MOON, LISA TEEL, AND WILLIAM J. RHEAD
}

Department of Pediatrics, University of Iowa, Iowa City, Iowa 52242

\begin{abstract}
We studied fibroblasts from three patients with long-chain acyl-coenzyme A dehydrogenase (LCADH) deficiency; siblings H.C. and J.C. had milder clinical phenotypes than unrelated patient R-1. In H.C., J.C., and R-1 oxidation of $\left[9,10(n)-{ }^{3} H\right]$ palmitate was 50 , 48 , and $28 \%$ of control, respectively, with $\mathrm{R}-1$ having significantly less activity than H.C. and J.C. $(p<0.05)$. Assays of mitochondrial short-chain and medium-chain acyl-coenzyme A dehydrogenases were normal in H.C. and J.C. However, mitochondrial LCADH activities in all three ranged from 17 to $21 \%$ of control. Flavin adenine dinucleotide addition increased LCADH activities in all three to 27-36\% of control. In the presence of monospecific medium-chain acyl-coenzyme $A$ dehydrogenase antiseria, LCADH activity decreased $17 \%$ in controls, and fell to $\leq 11 \%$ of control in J.C. and R-1. The heterogeneity observed in the $\left[{ }^{3} \mathrm{H}\right]$ palmitate oxidation studies was not explained by differences in LCADH activities under any assay condition. (Pediatr Res 23: 603-605, 1988)
\end{abstract}

\section{Abbreviations}

CoA, coenzyme A

LCADH, long-chain acyl-CoA dehydrogenase

LCD, long-chain acyl-CoA dehydrogenase deficiency

SCADH, short-chain acyl-CoA dehydrogenase

MCADH, medium-chain acyl-CoA dehydrogenase

FAD, flavin adenine dinucleotide

ETF, electron transfer flavoprotein

LCD has been identified in one of two siblings with nonketotic hypoglycemia and dicarboxylic aciduria (1) and in three patients with nonketotic hypoglycemia, carnitine deficiency, cardiomyopathy, and cardiorespiratory arrests associated with fasting (2, 3). LCD differs somewhat from other inborn errors of fatty acid metabolism (4-7) in that accumulated long-chain acyl-CoA produced a medium and long-chain $\left(\mathrm{C}_{6}-\mathrm{C}_{14}\right)$ dicarboxylic aciduria in one patient (8). The inability of skeletal and cardiac muscle to oxidize long-chain fatty acyl-CoA presumably produces muscle weakness and cardiomyopathy in some patients (3). We report herein studies in fibroblasts from two siblings with LCD (H.C. and J.C.) (8) and compare them to a previously reported LCD

Received December 7, 1987; accepted February 2, 1988.

Correspondence and reprint requests to Dr. William J. Rhead, Department of Pediatrics, University of lowa, lowa City, IA 52242.

Supported by Grant DK33289 from the National Institutes of Health, Grants 5-297 and 1-876 from the National Foundation, March of Dimes, and a clinical research grant from the Muscular Dystrophy Association.

${ }^{1}$ Portions of this work appeared as abstracts in Pediatr Res 1983;17:206A and 1987;21:339A. patient (R-1) (3). The clinical phenotypes of H.C. and J.C. in infancy and childhood have been well documented (8). The LCADH deficiency of H.C. has been reported briefly (3); ADH studies have never been reported for J.C. At $20 \mathrm{yr}$ of age, H.C. has a mild skeletal myopathy and profound carnitine deficiency without cardiomyopathy. She has repeated episodes of myalgias, myoglobinuria, and greatly elevated serum creatine phosphokinase levels provoked by fasting, exercise, fat ingestion, or emotional stress. She has elevated circulating levels of long-chain acyl-carnitines. J.C., her 18-yr-old sister, avoids fatty foods, takes frequent small meals, and has had few episodes of hypoglycemia or muscle weakness since childhood (Roe C, personal communication). Patient R-1 presented with cardiorespiratory arrest, lethargy, hypertrophic cardiomyopathy, and carnitine deficiency (3). To explore possible explanations for this marked clinical heterogeneity, we performed $\left[9,10(\mathrm{n})-{ }^{3} \mathrm{H}\right]$ palmitate cell oxidation studies, and assayed LCADH in the presence and absence of added FAD and MCADH antiserum.

\section{METHODS}

$\left[9,10(\mathrm{n}){ }_{-}^{3} \mathrm{H}\right]$ palmitate $(380 \mathrm{mCi} / \mathrm{mmol})$ was obtained from Research Products International, Arlington Heights, IL. FAD and N-ethylmaleimide were purchased from Sigma Chemical Co., St. Louis, MO. Pure pig liver MCADH and ETF were gifts from Dr. Carole L. Hall, Georgia Institute of Technology, Atlanta, GA. The pure pig kidney $\mathrm{MCADH}$ used for antisera preparation was a gift from Dr. C. Thorpe, University of Delaware, Newark, DE. Skin fibroblasts were obtained from patients H.C., J.C., and R-1 (courtesy of P. Coates, Children's Hospital of Philadelphia, Philadelphia, PA ) and six normal male infants. Fibroblasts were cultured in Eagle's minimal essential medium, supplemented with $10 \%$ fetal bovine serum, $2 \mathrm{mM}$ glutamine, $140 \mu \mathrm{M}$ penicillin, and $86 \mu \mathrm{M}$ streptomycin. Fibroblasts were subcultured 1:4 every $2 \mathrm{wk}$ and the media changed weekly. Cells of passage $6-19$ were used in all experiments. ${ }^{14} \mathrm{C}$-labeled substrate oxidation to ${ }^{14} \mathrm{CO}_{2}$ by intact fibroblasts in suspension was performed as described earlier (9). Tritium release from $[9,10(\mathrm{n})$ ${ }^{3} \mathrm{H}$ ]palmitate $(380 \mathrm{mCi} / \mathrm{mmol}, 22 \mu \mathrm{M})$ from fibroblast monolayers was performed as described previously (10). Preparation of fibroblast mitochondrial sonic supernatants and dye reduction $\mathrm{ADH}$ and ETF assays are described elsewhere $(7,9)$. FAD and monospecific MCADH antisera were preincubated with mitochondrial supernatants at $30^{\circ} \mathrm{C}$ for $5 \mathrm{~min}$ before $\mathrm{ADH}$ assay. Rabbit antiserum was raised to pure pig kidney MCADH as described earlier (4). This antiserum preparation completely inhibited MCADH activity towards octanoyl-CoA in human fibroblast and mitochondrial sonicates, and pure pig liver and kidney MCADH (4) (other data not shown). The estimate of variance used is the SEM. Means were compared with the Student's $t$ test; all $p$ values given are two-tailed and compare the patients' data to the respective control values. 


\section{RESULTS}

In preliminary experiments, fibroblasts from H.C. oxidized [1$\left.{ }^{14} \mathrm{C}\right]$ butyrate, $\mathrm{L}-\left[2-{ }^{14} \mathrm{C}\right]$ leucine, and $\left[1,4-{ }^{14} \mathrm{C}\right]$ succinate normally (77-141\% of control levels; data available on request). However, $\left[1-{ }^{14} \mathrm{C}\right]$-palmitate oxidation was significantly decreased $(43 \%$ of control, $p<0.05$; data available on request), comparable to the results obtained by Hale et al. (3) with the cells of R-1. H.C., J.C., and R-1 oxidized $\left[9,10(\mathrm{n}){ }_{-}^{-} \mathrm{H}\right]$ palmitate at 50,48 , and $28 \%$ of control levels, respectively. Using this substrate, the metabolic defect of R-1 appeared more severe than that in J.C. and H.C. $(n=15 ; p<0.05)$ (Table 1$)$.

Mitochondrial LCADH activities in patients H.C., J.C., and R- 1 were low and identical at 21,17 , and $20 \%$ of control values, respectively $(p<0.01)$, whereas SCADH and MCADH activities were normal (Table 2). Inasmuch as low intramitochondrial FAD pools in vivo could result in deficient $\mathrm{ADH}$ activities, we added $20 \mu \mathrm{M}$ FAD to mitochondrial supernatants. LCADH activities increased significantly to $27-36 \%$ of control in all three patients (one tail $p=0.03$; Table 2). We also assayed mitochondrial MCADH and LCADH activities after incubation with monospecific rabbit antisera to pig kidney $\mathrm{MCADH}$, which completely inhibits human fibroblast MCADH activity (4) and permits accurate assay of LCADH activity alone (Table 2). Under our assay conditions, $17 \%$ of palmityl-CoA dehydrogenation in normal fibroblasts appeared due to MCADH activity. LCADH activities in patients J.C. and R-1 fell to 6 and $11 \%$ of control, respectively.

In all three patients, ETF activities were normal $(\geq 74 \%$ of control, $p>0.5$ ), making it unlikely that any represent an ETFdeficient multiple acyl-CoA dehydrogenation disorder variant (Table 3).

Table 1. $\left[9,10(n)-{ }^{3} H\right]$ Palmitate oxidation by intact fibroblasts $(\mathrm{nmol} / \mathrm{mg} \text { protein } / \mathrm{h} \pm S E M)^{*}$

Cell line

$\begin{array}{ll}\text { Normal controls: } & \\ 1 & 0.37 \pm 0.05 \\ 2 & 0.43 \pm 0.04 \\ \text { H.C. } & 0.20 \pm 0.04 \dagger \\ \text { J.C. } & 0.19 \pm 0.02 \dagger \\ \text { R-1 } & 0.11 \pm 0.03 \dagger\end{array}$

* Cells from H.C., J.C., and R-1 and two controls were assayed simultaneously under identical conditions in 15 experiments as described previously (10). $p$ values refer to comparisons between patient and control data.

$\dagger p<0.01$.

\section{DISCUSSION}

We describe herein detailed studies in fibroblasts from three patients with LCD. The radiolabeled substrate oxidation studies revealed defective long-chain fatty acid oxidation in all three patients. Preliminary $\left[{ }^{14} \mathrm{C}\right]$ substrate oxidation studies revealed no significant differences between H.C. and R-1, two patients with widely varying clinical phenotypes. However, patient R-1 catabolized $\left[{ }^{3} \mathrm{H}\right]$ palmitate less well than either H.C. or J.C. $(p<$ $0.05)$, consistent with their differing clinical courses.

ADH assays in mitochondrial sonicates revealed low $\mathrm{LCADH}$ activites in all three patients ( $\leq 21 \%$ of control), whereas $\mathrm{MCADH}$ and SCADH activities were normal. In our assay system, the residual activities with palmityl-CoA were somewhat higher in R-1 than those reported by Hale et al. (3) and higher than anticipated for isolated LCADH deficiency. This observation may result from overlapping substrate specificities of human fibroblast $\mathrm{ADH}$, as reported for the bovine enzymes (11). In many species, three different enzymes dehydrogenate straightchain fatty acids; LCADH is active with acyl-CoA of 8-22 carbons, MCADH, 4-12 carbons, and SCADH, 4-6 carbons (11, 12). Whereas the chain length specificities of human $\mathrm{ADH}$ are not known, human fibroblast MCADH can dehydrogenate a broad range of acyl-CoA. In two patients with SCADH deficiency, apparent SCADH activities were $\leq 53 \%$ of controls, but fell to $\leq 11 \%$ of control after addition of MCADH monospecific antisera (4). The apparent SCADH activities resulted from MCADH acting on butyryl-CoA, the optimal substrate for SCADH. MCADH-deficient patients also have low SCADH activities (42 to $73 \%$ of control) $(5,13)$ reflecting the loss of MCADH activity toward butyryl-CoA. MCADH-deficient patients also have LCADH activities 79-96\% of control, suggesting MCADH also dehydrogenates palmityl-CoA. Herein addition of

Table 3. ETF activities in fibroblasts [pmol/min/mg protein \pm $\operatorname{SEM}(n)]^{*}$

\begin{tabular}{lcc}
\hline & \multicolumn{2}{c}{ Cell Preparation } \\
\cline { 2 - 3 } \multicolumn{1}{c}{ Cell line } & $\begin{array}{c}\text { Mitochondrial } \\
\text { supernatants }\end{array}$ & $\begin{array}{c}\text { Fibroblast } \\
\text { supernatants }\end{array}$ \\
\hline Normal controls & $460 \pm 54(2)$ & $80 \pm 20(6)$ \\
H.C. & $\mathrm{ND} \dagger$ & $59 \pm 11(3)$ \\
J.C. & $514(1)$ & ND \\
R-1 & $353(1)$ & ND \\
\hline
\end{tabular}

* Activities were determined as previously described (7). The number of determinations totaled two to six using four control lines and one to three for each patient line.

$\dagger$ Not determined.

Table 2. ADH activities in fibroblast mitochondrial sonic supernatants [pmol/min/mg protein $\pm S E M(n)]^{*}$

\begin{tabular}{|c|c|c|c|c|c|c|c|}
\hline \multirow[b]{2}{*}{ Cell line } & \multirow{2}{*}{$\begin{array}{l}\text { Enzyme assayed: } \\
\text { Substrate: } \\
\text { Additions: }\end{array}$} & \multirow{2}{*}{$\begin{array}{c}\text { MCADH } \\
\text { Octanoyl-CoA } \\
\text { SCADH } \\
\text { Butyryl-CoA } \\
\text { None }\end{array}$} & \multicolumn{2}{|c|}{$\begin{array}{c}\text { MCADH } \\
\text { Octanoyl-CoA }\end{array}$} & \multicolumn{3}{|c|}{$\begin{array}{c}\text { LCADH } \\
\text { Palmityl-CoA }\end{array}$} \\
\hline & & & None & $\begin{array}{l}\text { MCADH } \\
\text { antiserum }\end{array}$ & None & FAD $(20 \mu \mathrm{M})$ & $\begin{array}{l}\text { MCADH } \\
\text { antiserum }\end{array}$ \\
\hline Normal controls & & $830 \pm 96(10)$ & $1580 \pm 115(10)$ & $0.0 \pm 0.0(10)$ & $790 \pm 102(10)$ & $805 \pm 95(10)$ & $656 \pm 70(9)$ \\
\hline H.C. & & $639 \pm 52(6)$ & $1469 \pm 120(8)$ & $\mathrm{ND} \dagger$ & $166 \pm 20(14) \ddagger$ & $290 \pm 32(6) \ddagger$ & ND \\
\hline J.C. & & $672 \pm 41(2)$ & $1185 \pm 40(2)$ & ND & $134 \pm 31(3) \ddagger$ & $217 \pm 36(3) \ddagger$ & $39 \pm 15(3) \S$ \\
\hline $\mathrm{R}-1$ & & ND & ND & ND & $158 \pm 45(3) \ddagger$ & $242 \pm 41(3) \ddagger$ & $72 \pm 18(4) \S$ \\
\hline
\end{tabular}

* ADH activities were assayed by the dye reduction methods described earlier $(4,5)$. The number of determinations totaled nine to 10 using six control lines and two to 11 for each patient line; in all experiments, at least two control lines were assayed simultaneously with patient lines. $p$ values refer to comparisons between patient and control data.

$\dagger$ Not determined.

$\ddagger p<0.01$.

$\S p<0.001$. 
monospecific MCADH antisera revealed LCADH activities to be $\leq 11 \%$ of control in J.C. and R-1.

All three straight chain ADH bind FAD ionically (12). As purified rat liver $\mathrm{LCADH}$ apoenzyme is converted to its holenzyme after incubation with FAD (12), we preincubated control and patient mitochondrial sonicates with this cofactor to maximize holoenzyme formation. Control LCADH activity increased little, whereas LCADH activities increased in all patients. This in vitro phenomenon may not reflect a physiologically significant FAD response, however, riboflavin supplementation might merit a therapeutic trial in $L C D$ patients.

Whereas patient R-1 had a more severe clinical course than H.C., who had more episodes than her sibling, J.C., all three have a similar degree of enzymatic deficiency in vitro, both in the presence and absence of FAD and MCADH antiserum. However, our $\left[{ }^{3} \mathrm{H}\right]$ palmitate oxidation results parallel the clinical severities in these three patients and suggest that other biochemical mechanisms improve $\beta$-oxidation efficiency in H.C. and J.C. Relevant possibilities include an altered LCADH with significantly greater activity in vivo than in disrupted cells or mitochondria. However, inasmuch as our $\left[{ }^{3} \mathrm{H}\right]$ palmitate oxidation assay does not distinguish between mitochondrial and peroxisomal $\beta$-oxidation, the differences in $\left[{ }^{3} \mathrm{H}\right]$ palmitate oxidation seen in these patients could be due to increased activity of peroxisomal $\beta$-oxidation in the siblings H.C. and J.C. We did not perform complementation analyses (10) between these three patients because all biochemical analyses suggest that all have the same enzymatic defect. Further study of these and other LCD patients should improve our understanding of molecular and biochemical variations of $\beta$-oxidation in man.

Acknowledgments. The authors thank Drs. C. Hall and C. Thorpe for the gifts of MCADH and ETF, P. Coates and D. Hale for R-1 cells, and Ms. Linda Abel for clerical assistance.

\section{REFERENCES}

1. Amendt BA, Fritchman KN, Rhead WJ 1983 Dicarboxylic aciduria (DCA) due to deficiency of the long chain acyl-CoA dehydrogenase (LCADH) reversible by addition of flavin adenine dinucleotide (FAD). Pediatr Res 17:206A

2. Hale D, Coates P, Stanley C, Cortner J, Hall C 1983 Long chain acyl-CoA dehydrogenase deficiency. Pediatr Res 17:290A

3. Hale DE, Batshaw ML, Coates PM, Frerman FE, Goodman SE, Singh I, Stanley CA 1985 Long-chain acyl coenzyme A dehydrogenase deficiency: an inherited cause of nonketotic hypoglycemia. Pediatr Res 19:666-671

4. Amendt BA, Greene C, Sweetman L, Cloherty J, Shih V, Moon A, Teel L, Rhead WJ 1987 Short chain acyl-CoA dehydrogenase deficiency: clinical and biochemical studies in two patients. Clin Invest 79:1303-1309

5. Amendt BA, Rhead WJ 1985 Catalytic defect of medium chain acyl-coenzyme A dehydrogenase deficiency. Lack of both cofactor responsiveness and biochemical heterogeneity in eight patients. Clin Invest 76:963-969

6. Gregersen N 1985 The acyl-CoA dehydrogenation deficiencies. J Clin Lab Invest 45(suppl 174):1-60

7. Amendt BA, Rhead WJ 1986 The multiple acyl-coenzyme A dehydrogenation disorders, glutaric aciduria type II and ethylmalonic-adipic aciduria. J Clin Invest 78:205-213

8. Naylor EW, Mosovich LL, Guthrie R, Evans JE, Tieckelmann H 1980 Intermittent non-ketotic dicarboxylic aciduria in two siblings with hypoglycemia: an apparent defect in B-oxidation of fatty acids. J Inherited Metab Dis 3:1924

9. Rhead W, Amendt B, Fritchman K, Felts S 1983 Dicarboxylic aciduria: deficient $\left[1-{ }^{14} \mathrm{C}\right]$ octanoate oxidation and medium chain acyl-CoA dehydrogenase activity in fibroblasts. Science 221:73-75

10. Moon A, Rhead WJ 1987 Complementation analysis of fatty acid oxidation disorders. J Clin Invest 79:59-64

11. Davidson B, Schulz H 1982 Separation properties, and regulation of acyl coenzyme A dehydrogenases from bovine heart and liver. Arch Biochem Biophys 213:155-162

12. Ikeda Y, Okamura-Ikeda K, Tanaka K 1985 Purification and characterization of short-chain, medium-chain and long-chain acyl-CoA dehydrogenases from rat liver mitochondria. J Biol Chem 260:1311-1325

13. Coates PM, Hale DE, Stanley CA, Corkey BE, Cortner JA 1985 Genetic deficiency of medium-chain acyl-coenzyme A dehydrogenase: studies in cultured skin fibroblasts and peripheral mononuclear leukocytes. Pediatr Res 19:671-676 